
\title{
R Reserarch Soure \\ The use of 3D printing pelvis and hip in a complex THA: clinical experience and reports of literature
}

\author{
Chenyu Huang \\ Nanjing First Hospital \\ Qingqiang Yao \\ Nanjing First Hospital \\ Wei Liu \\ Nanjing Medical University \\ Yan Xu \\ Nanjing First Hospital \\ Cheng Tang \\ Nanjing First Hospital \\ Bo Wei \\ Nanjing First Hospital \\ Jiayi Li \\ Nanjing First Hospital \\ Liming Wang ( $\square$ Imwang@njmu.edu.cn ) \\ Nanjing First Hospital
}

\section{Research}

Keywords: 3D printing, Crowe type IV DDH, THA, Addictive manufacturing

Posted Date: December 5th, 2020

DOI: https://doi.org/10.21203/rs.3.rs-52917/v2

License: (c) (1) This work is licensed under a Creative Commons Attribution 4.0 International License.

Read Full License 


\section{Abstract}

Background: Total hip arthroplasty(THA) is a good solution of developmental dysplasia of the hip(DDH). However, patients diagnosed with Crowe type IV are difficult to deal with. With traditional operation skills, the operation time is quite long and the patients will lose a lot of blood.

Case presentation: We present the case of a 58-year-old male patient who underwent left THA due to Crowe type IV DDH with the help of 3D printing models. Before the operation, we put the CT image into a computer software named mimics. After getting the stl. document, we use 3D printer to print the models out. By using the models, we planned the operation in advance. And after sterilizing the models, we put them in the operation area, which saves a lot of operation time and reduces much blood loss by giving surgeons a better anatomy view.

Conclusion: THA with the help of 3D printing pelvis and femur can result in a relatively short recovery time and provide good clinical efficacy of type IV DDH patients.

\section{Background}

Developmental dysplasia of the hip (DDH) is a condition characterized by morphological abnormalities of the acetabulum and femur. And surgeons classified it according to dislocation severity, from I to IV, most commonly based on the Crowe rubric. And the type IV is the most severe of the four types[1]. Primary total hip arthroplasty (THA) is complicated and difficult in patients with Crowe type IV (type IV from here forward for simplicity) DDH because the pathological anatomical changes that accompany this condition. Compared to traditional THA, the operation time is longer and intraoperative bleeding is much more.

In the 1970s, John Charnley introduced the trochanteric osteotomy for complicated primary hip replacement, including in patients with type IV DDH[2]. Along with the good performance of the patients after the operations, is the longer operation time and more intraoperative bleeding

Some Crowe type IV DDH patients undergoing THA require a femoral shortening osteotomy to equalize the length of the lower extremities and decrease the difficulty of intraoperative reduction and the incidence of complications [3-5]. The femoral subtrochanteric transverse osteotomy is one of the most common forms of osteotomy [6, 7].

Although several such surgical protocols have been reported to yield excellent results, each has drawbacks. Thus it is necessary to invent a method to save operation time and reduce intraoperative bleeding.

With the development of the 3D printing technique, 3D printing pelvis and femur can be of great use in the surgeries of THA. Our study reports the case of a patient with type IV DDH who underwent THA with 
the help of 3D printing pelvis and femur. The time and blood control of the surgery and recovery time after THA are discussed based on literature reports.

\section{Case Presentation}

The patient we operated on is a 58-year-old male, who came to our hospital because of the limited activity of the left hip. He suffers from the intermittent pain of the hip for more than 40 years and the pain aggravated for 6 months. We X-rayed his pelvis and found that his left hip was dislocated(Fig. 1). Then he was diagnosed with Crowe type IV with a Harris score of 23 and visual analogue scale (VAS) score of 8. After the physical examination, we found that the patient's left leg is shorted by $4.2 \mathrm{~cm}$ and he suffers from the pain of hip rotation. The degrees of right hip joint motion were as follows: adduction, $10^{\circ}$; internal rotation $10^{\circ}$; and external rotation, $10^{\circ}$; flexion, $80^{\circ}$; and outreach, $15^{\circ}$. There is no other diseases reported by the patient.

First, we collected the patients' image data by using MRI(Siemens, Berlin, Germany) and CT (Philips, Eindhoven, Netherlands) plain scans. Then we import the MRI and CT data (DICOM format) for the surgical area into the computer and reconstructed it via Mimics 21.0 software (Materialize, Leuven, Belgium). We used different colors to distinguish different parts of the structure and finally we got a 3D digital model. (Fig. 2). After getting the 3D digital model, we analyze the condition of the patients and developed a surgical plan, which helped us get to know the patients better. And then we use 3D printer to print the model out and sterilize the 3D model. (Fig. 3).

We performed the surgery through the posterior-lateral approach. The patient was placed in the right lateral position after sciatic nerve block and lumbar plexus anesthesia. We made a roughly $12-\mathrm{cm}$ posterolateral incision on the left hip of the patient right after disinfection and draping. And the epidermis and subcutaneous tissue of the surgical area were dissected to expose the selected bony landmark according to the surgical plan. First, we compared the sterilized 3D model with the patient's structure. Second, in the preoperative design of the acetabulum cup position, we use the acetabular reamer with a diameter of $36 \mathrm{~mm}$ to grind to a diameter of $46 \mathrm{~mm}$ (Fig. 4A), and then we inserted a $48 \mathrm{~mm}$ diameter cementless acetabular cup and reinforced it with two acetabular screws (Fig. 4B). Time to time, we used the 3D printing model to avoid extra procedures. And then we opened the femoral medullary cavity enlarged it to the size of 10.5 with the medullary cavity file(Fig. 4C). We installed the femoral prosthesis with a 36mm diameter short neck head. At last, we reset the hip joint and checked its motion. (Fig. 4D). Before suturing layer by layer, we placed a drainage tube deep into the surgical area. The operation lasted for $2 \mathrm{~h}$, which is much quicker than traditional DDH surgery. Also with the help the 3D printing models, the intraoperative bleeding is less than usual.

To prevent infection, the patient received antibiotics right after the operation. And he toke the X-ray on the second day of the surgery, which shows good position of the implants.

\section{Discussion}


The other name of three-dimensional printing (3DP) is rapid prototyping, which constracts 3D models layer by layer. It is an additive manufacturing process that promote the production of the complex geometrical. The development of imaging modalities and the 3D printing technology enables a big advancement in the use of 3D printing models of some operation practices. $(10,11)$.

Nowadays, the use of 3D printing technology in clinical practice focuses on 3D printing models(12-15). Previous researchers made a lot of researches(16-18) in virtual and haptic patient specific anatomical models, often termed biomodels (19). It can help surgeons to plan the operations in advance and save their operation time. So we operated this special operation:3D printing models assisted complex THA.

According to the previous researches, surgeons all around the world hold the opinion that the 3D printing models are quite beneficial when they are operating difficult operations. [20] Previously, before and during the operations, most of the surgeons relied on two-dimensional (2D) images, for example, X-ray, CT and/ or MRI. And the 1:1 scale 3D printing models could provide the surgeons with the better understanding of the anatomy compared to the $2 \mathrm{D}$ visualization.

Also, before the operations, we can sterilize these models. In that way, surgeons could use these models to get a template to review intra-operatively[21,22]. However, the work people have done on the models is limited. Previous researches have found that, compared to traditional operations, the application of 3D printing models can offer more accurate evaluation of relevant surgical structures in complex cases, for example, acetabular fractures and spinal deformities.[22]

Besides the subjective benefits, we can also find some other objective advantages of the 3D printed models. Researchers found that compared to traditional operations, the surgery time, intraoperative blood loss and fluoroscopy time are much less[23-30], which improves the quality of planned placement of implants and selection of instrumentation. And thus the need to contour and the implants number of adjustments intraoperatively are reduced.

Nowadays, surgeon often use subtrochanteric shortening osteotomy to deal with the type IV DDH. And in our study, with the usage of the 3D printing models, the surgery time, intraoperative blood loss and fluoroscopy time are much less, which is of great benefit.

Disadvantages are also obvious. First is the cost. For better evaluation, we have to compare the potential savings of the improved operations and production cost of the 3D printing models. Second is that the 3D printing model don't include the soft tissue structures, as well as the nerves and blood vessels. To better serve the clinical practice, further studies and a streamlined protocol for the 3D printing models are needed in the future.

\section{Conclusion}

THA with the help of 3D printing pelvis and femur can result in a relatively short recovery time and provide good clinical efficacy of type IV DDH patients. 


\section{Abbreviations}

3D: Three-dimensional; DDH: Developmental dysplasia of the hip; CT: Computed tomography; THA: Total hip arthroplasty

\section{Declarations}

\section{Acknowledgements}

Not applicable.

\section{Authors' contributions}

$\mathrm{CYH}$ and QQY contributed to the study design and the drafting of the article. WL, CT and BW collected CT data and CAD. JYL, HKZ and CYH cleaned, prepared and sterilized the model. JTL and LMW contributed to all surgical procedures. The authors read and approved the final manuscript.

\section{Funding}

National Natural Science Foundation of China(81771985)

\section{Availability of data and materials}

The datasets used and/or analyzed during the current study are available from the corresponding author on reasonable request.

\section{Ethics approval and consent to participate}

All procedures performed in studies involving human participants were in accordance with the ethical standards of the institutional and/or national research committee and with the 1964 Helsinki Declaration and its later amendments or comparable ethical standards. Informed consent was obtained from all individual participants included in the study.

\section{Consent for publication}

Not applicable.

\section{Competing interests}

The authors declare that they have no competing interests.

\section{References}

1. Crowe JF, Mani VJ, Ranawat CS. Total hip replacement in congenital dislocation and dysplasia of the hip. J Bone Joint Surg Am, 1979, 61: 15-23. 
2. Charnley J. Total hip replacement by low-friction arthroplasty. Clin Orthop Relat Res, 1970, 72: 7-21.

3. Krych AJ, Howard JL, Trousdale RT, Cabanela ME, Berry DJ. Total hip arthroplasty with shortening subtrochanteric osteotomy in Crowe type-IV developmental dysplasia. J Bone Joint Surg Am. 2009;91(9):2213-21.

4. Wang D, Li LL, Wang HY, Pei FX, Zhou ZK. Long-Term Results of Cementless Total Hip Arthroplasty with Subtrochanteric Shortening Osteotomy in Crowe Type IV Developmental Dysplasia. J Arthroplast. 2017;32(4):1211-9.

5. Ollivier M, Abdel MP, Krych AJ, Trousdale RT, Berry DJ. Long-Term Results of Total Hip Arthroplasty with Shortening Subtrochanteric Osteotomy in Crowe IV Developmental Dysplasia. J Arthroplast. 2016;31(8):1756-60.

6. Park MS, Kim KH, Jeong WC. Transverse subtrochanteric shortening osteotomy in primary total hip arthroplasty for patients with severe hip developmental dysplasia. J Arthroplast. 2007;22(7):1031-6.

7. Muratli KS, Karatosun V, Uzun B, Celik S. Subtrochanteric shortening in total hip arthroplasty: biomechanical comparison of four techniques. J Arthroplast. 2014;29(4):836-42.

8. Sofu H, Kockara N, Gursu S, Issin A, Oner A, Sahin V. Transverse subtrochanteric shortening osteotomy during cementless total hip arthroplasty in Crowe type-III or IV developmental dysplasia. J Arthroplasty, 2015, 30: 1019-1023.

9. Mu W, Yang D, Xu B, Mamtimin A, Guo W, Cao L. Midterm outcome of cementless total hip arthroplasty in Crowe IV-Hartofilakidis type III developmental dysplasia of the hip. J Arthroplasty, 2016, 31: 668-675.

10. Rengier F, Mehndiratta A, Von Tengg-Kobligk H, et al.3D printing based on imaging data: review of medical applications. Int J Comput Assist Radiol Surg 2010;5:335-41.

11. Wilcox B, Mobbs RJ, Wu AM, et al. Systematic review of 3D printing in spinal surgery: the current state of play. J Spine Surg 2017;3:433-43.

12. Kim J, Rajadurai J, Choy WJ, et al. Three-Dimensional Patient-Specific Guides for Intraoperative Navigation for Cortical Screw Trajectory Pedicle Fixation. World Neurosurg 2019;122:674-9.

13. Phan K, Sgro A, Maharaj MM, et al. Application of a 3D custom printed patient specific spinal implant for C1/2 arthrodesis. J Spine Surg 2016;2:314-8.

14. Lu S, Xu YQ, Lu WW, et al. A novel patient-specific navigational template for cervical pedicle screw placement. Spine 2009;34:E959-E966.

15. D'Urso PS, Williamson OD, Thompson RG. Biomodeling as an Aid to Spinal Instrumentation. Spine 2005;30:2841-5.

16. Burnard JL, Parr WCH, Choy WJ, et al. 3D-printed spine surgery implants: a systematic review of the efficacy and clinical safety profile of patient-specific and off-the-shelf devices. Eur Spine J 2019. [Epub ahead of print]. 
17. Choy WJ, Mobbs RJ, Wilcox B, et al. Reconstruction of Thoracic Spine Using a Personalized 3DPrinted Vertebral Body in Adolescent with T9 Primary Bone Tumor. World Neurosurg 2017; 105:1032.e13-1032.e17.

18. Choy WJ, Parr WCH, Phan K, et al. 3-dimensional printing for anterior cervical surgery: a review. J Spine Surg 2018;4:757-69.

19. D'Urso PS, Askin G, Earwaker JS, et al. Spinal Biomodeling. Spine 1999;24:1247-51.

20. Jiang Michael,Chen Gordon,Coles-Black Jasamine et al. Three-dimensional printing in orthopaedic preoperative planning improves intraoperative metrics: a systematic review.[J] .ANZ J Surg, 2019,

21. Bizzotto N, Tami I, Santucci A et al. 3D printed replica of articular fractures for surgical planning and patient consent: a two years multi- centric experience. 3D Print. Med. 2015; 2: 2.

22. Jentzsch T, Vlachopoulos L, Furnstahl P, Muller DA, Fuchs B. Tumor resection at the pelvis using three-dimensional planning and patient- specific instruments: a case series. World J. Surg. Oncol. 2016; 14: 249.

23. Chen C, Cai L, Zhang C, Wang J, Guo X, Zhou Y. Treatment of diepunch fractures with 3D printing technology. J. Invest. Surg. 2017; 31: 385-92.

24. Yang L, Grottkau B, He Z, Ye C. Three dimensional printing technology and materials for treatment of elbow fractures. Int. Orthop. 2017; 41: 2381-7

25. Yang L, Shang XW, Fan JN et al. Application of 3D printing in the surgical planning of Trimalleolar fracture and doctor-patient communica- tion. Biomed. Res. Int. 2016; 2016: 2482086.

26. Li C, Yang M, Xie Y et al. Application of the polystyrene model made by 3D printing rapid prototyping technology for operation planning in revision lumbar discectomy. J. Orthop. Sci. 2015; 20: 475-80.

27. Zheng SN, Yao QQ, Mao FY et al. Application of 3D printing rapid prototyping-assisted percutaneous fixation in the treatment of inter- trochanteric fracture. Exp. Ther. Med. 2017; 14: 3644-50.

28. Karlin L, Weinstock P, Hedequist D, Prabhu SP. The surgical treatment of spinal deformity in children with myelomeningocele: the role of per- sonalized three-dimensional printed models. J. Pediatr. Orthop. B 2017; 26: 375-82.

29. Yang M, Li C, Li Y et al. Application of 3D rapid prototyping technology in posterior corrective surgery for Lenke 1 adolescent idiopathic scoliosis patients. Medicine 2015; 94: e582.

30. Cherkasskiy L, Caffrey JP, Szewczyk AF et al. Patient specific 3D print models improve deformity correction after proximal femoral osteotomy for slipped capital femoral epiphysis. J. Invest. Med. 2016; 64: 197.

\section{Figures}




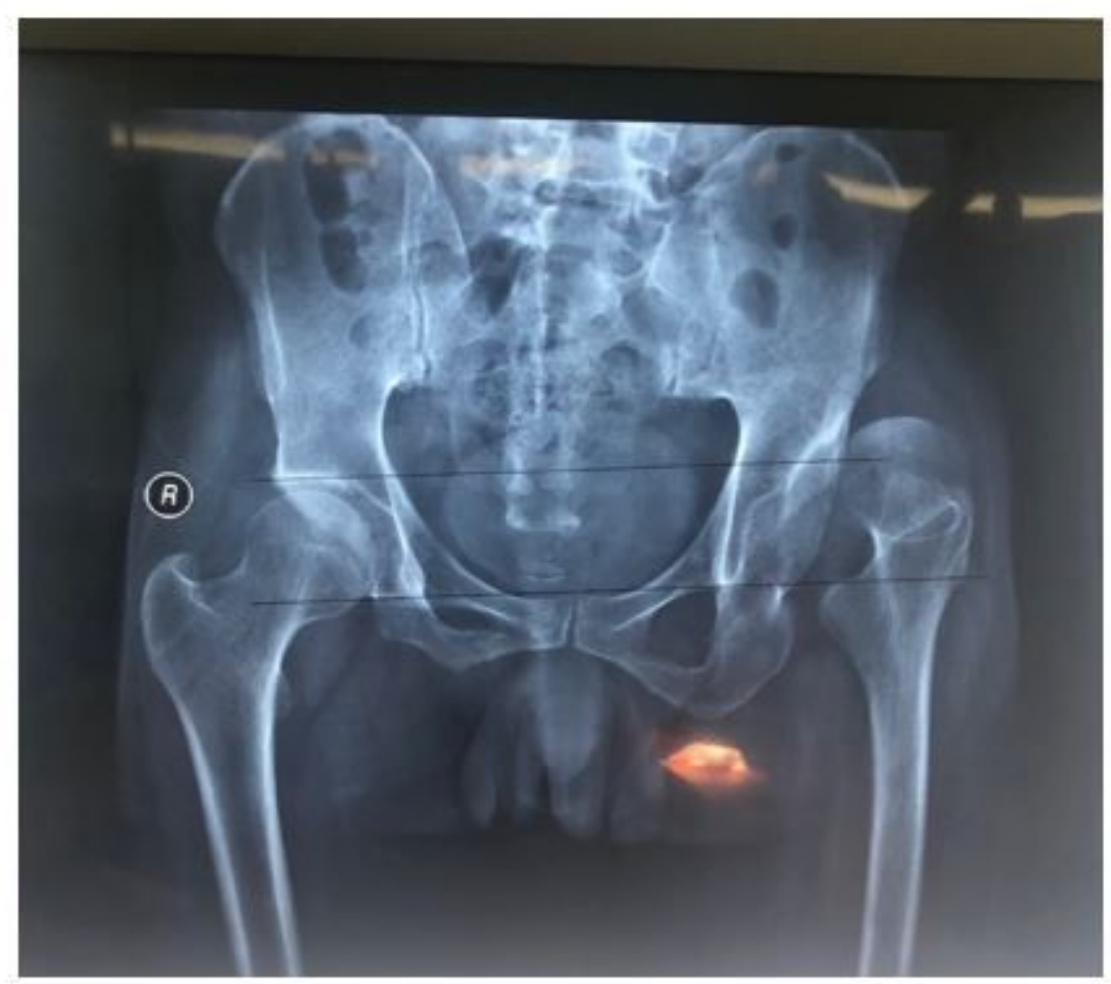

Figure 1

Patient X-ray image before the operation
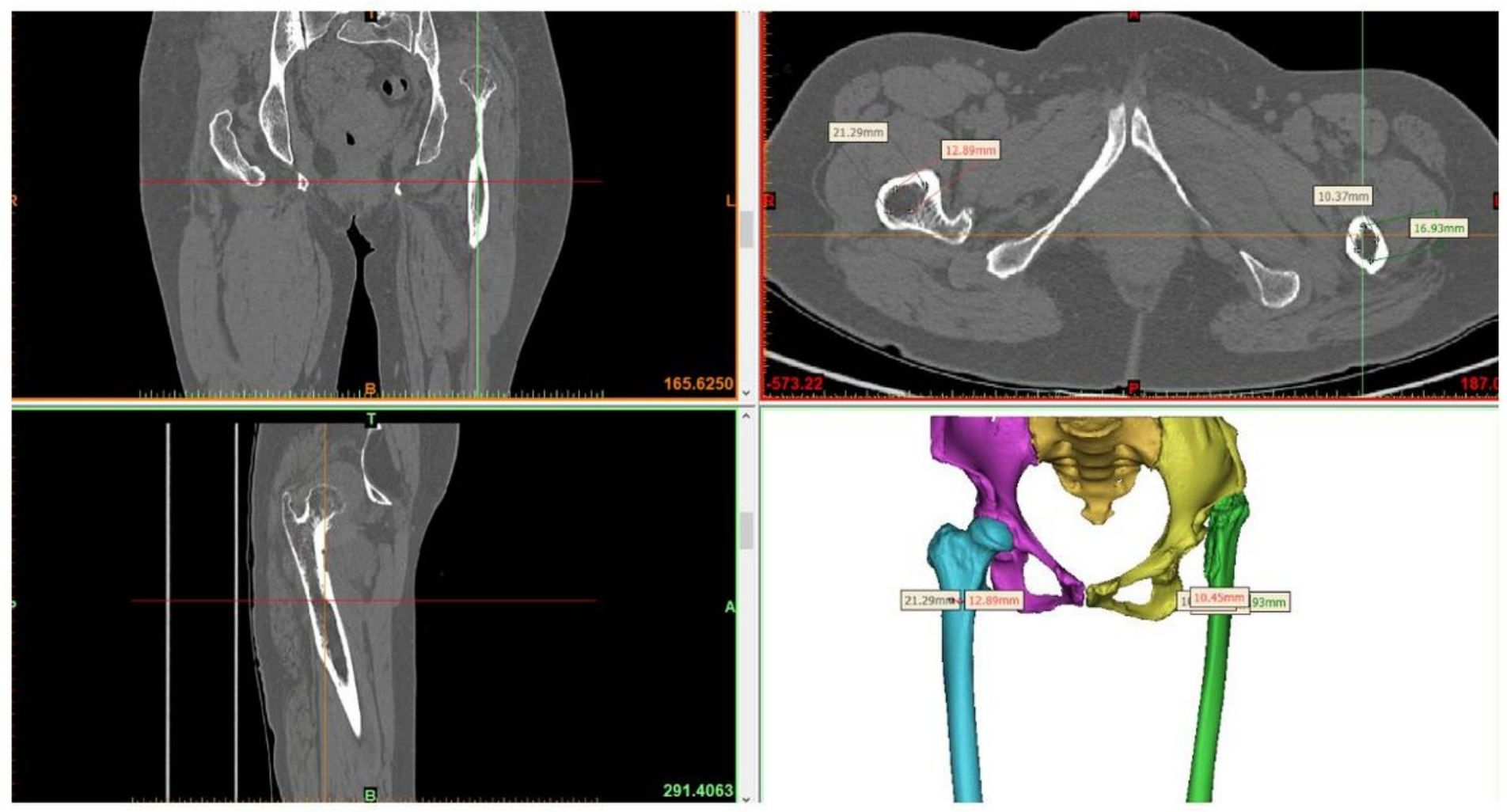
Figure 2

3D digital model of the patient
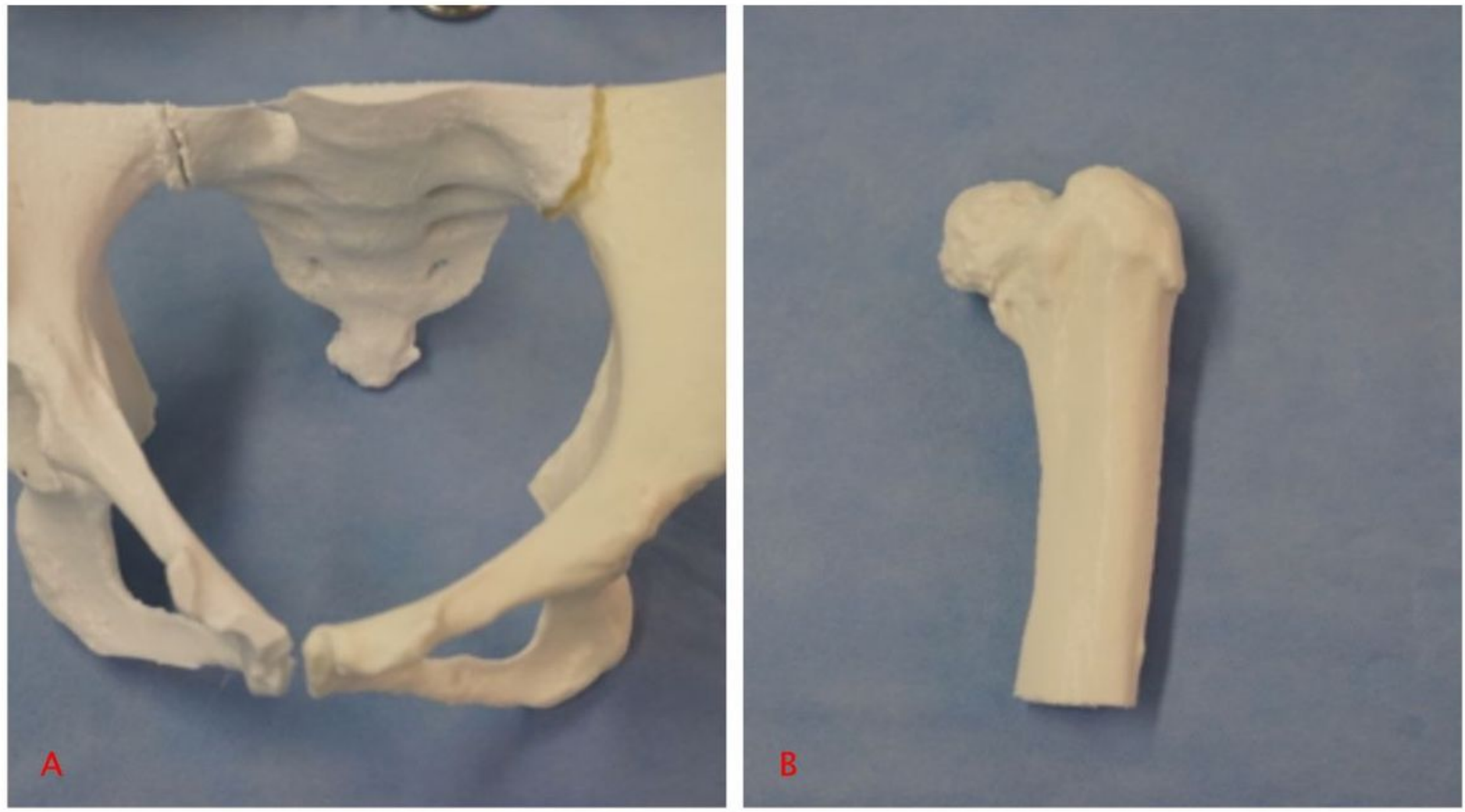

Figure 3

3D printed models 

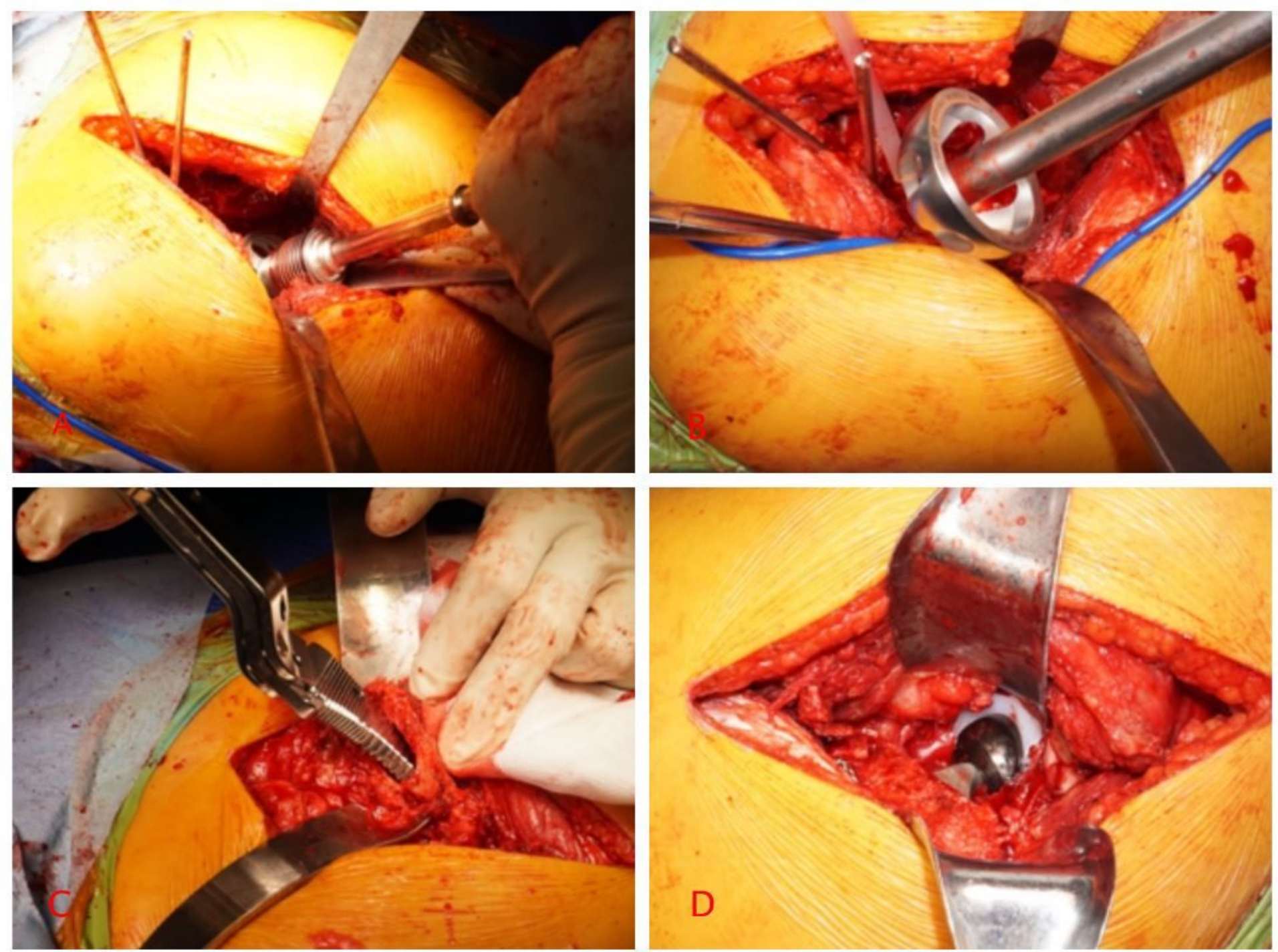

Figure 4

Procedure of the surgery 